\title{
Natural flavonoids as antidiabetic agents. The binding of gallic and ellagic acids to glycogen phosphorylase $b$
}

Efthimios Kyriakis, George A. Stravodimos, Anastassia L. Kantsadi, Demetra S.M. Chatzileontiadou, Vassiliki T. Skamnaki, and Demetres D. Leonidas*

Department of Biochemistry and Biotechnology, University of Thessaly, 26 Ploutonos Str. 41221 Larissa, Greece

* To whom correspondence should be addressed:

Assoc. Prof. Demetres D. Leonidas, Department of Biochemistry and Biotechnology, University of Thessaly, 26 Ploutonos Str. 41221 Larissa, Greece. Phone: +30 2410 565278; Fax: +30 2410 565290; e-mail: ddleonidas@bio.uth.gr. 


\section{Abstract}

We present a study on the binding of gallic acid and its dimer ellagic acid to glycogen phosphorylase (GP). Ellagic acid is a potent inhibitor with $K_{\mathrm{i}} \mathrm{S}$ of 13.4 and $7.5 \mu \mathrm{M}$, in contrast to gallic acid which displays $K_{\mathrm{i}} \mathrm{S}$ of 1.7 and $3.9 \mathrm{mM}$ for $\mathrm{GPb}$ and $\mathrm{GPa}$, respectively. Both compounds are competitive inhibitors with respect to the substrate, glucose-1-phoshate, and non-competitive to the allosteric activator, AMP. However, only ellagic acid functions with glucose in a strongly synergistic mode. The crystal structures of the GPb-gallic acid and GPb-ellagic acid complexes were determined at high resolution, revealing that both ligands bind to the inhibitor binding site of the enzyme and highlight the structural basis for the significant difference in their inhibitory potency. 
Keywords: flavonoids; ellagic acid; gallic acid; glycogen phosphorylase; type 2 diabetes; enzyme inhibition; X-ray crystallography.

Abbreviations: GP, glycogen phosphorylase; GPb, rabbit muscle glycogen phosphorylase b; PLP, pyridoxal 5'-phosphate; Glc-1-P, $\alpha$-D-glucose 1-phosphate; r.m.s.d., root-mean-square displacement;

\section{Highlights:}

- Ellagic acid is the most potent inhibitor of natural origin that binds at the glycogen phosphorylase inhibitor site.

- Gallic acid is a weak inhibitor that also binds at the inhibitor site of glycogen phosphorylase

- Ellagic acid's binding interacts strongly with glucose binding to GPb whereas gallic acid's does not. 


\section{Introduction}

Glycogen phosphorylase (GP; E.C. 2.4.1.1) is the most well studied enzyme in glycogen metabolism [1]. It catalyses the first step in the intracellular degradation of glycogen to yield Glc-1-P. Because of its central role in glucose homeostasis, GP has been exploited for the discovery of potent and specific inhibitors [2-4] which may be used as antihyperglycaemic agents. In the last decade, numerous studies have validated GP as a pharmaceutical target for type 2 diabetes [5]. GP is an allosteric enzyme that follows the MWC model [6]. It exists in two interconvertible by phosphorylation forms: GPb (unphosphorylated, T state, low activity and substrate specificity) and GPa (phosphorylated, R state, high activity and substrate specificity) [1]. GPb is a homodimer with six distinct binding sites, the catalytic, the inhibitor, the allosteric, the glycogen storage, the indole-binding and the quercetin binding sites $[1,7]$. The molecular basis of the allosteric transition from the $T$ to $R$ state lies with a flexible loop (280s, residues 282-286) which in the $T$ state partially blocks access to the catalytic site. Upon transition from the $T$ to $R$ state, this loop becomes disordered, allowing Arg569 to enter the catalytic site and create the phosphate recognition site; that also provides access for the substrate (glycogen) to reach the catalytic site [1].

The majority of the GP reported inhibitors are glucose derivatives $[2-4,8]$ that bind to the catalytic site and compete for binding with the substrate. The catalytic site of $\mathrm{GPb}$ is buried at the center of the protein molecule and it is accessible to the bulk solvent through a $15 \AA$ long channel. At the entrance of this channel, on the surface of the enzyme and approximately $12 \AA$ from the catalytic site, is the inhibitor site [1]. In the T-state, two hydrophobic residues, Phe285 from the 280s loop, and Tyr613, are stacked together to form the inhibitor site. The inhibitor site shows great binding diversity, but it mainly binds purines, flavonoids, and their derivatives such as IMP, ATP, NADH, FMN and FAD [1-4]. A ligand, on binding at this site, stabilizes the T-state conformation and inhibits the enzyme by blocking the channel that provides access to the catalytic site. The physiological significance of the inhibitor site has yet to be established but it may be used by an unidentified compound to enhance the effects of the control of liver GP by glucose, possibly in response to insulin $[9,10]$.

In the recent years, GP has been used for the screening of natural products [4] for the discovery of nonprescription nutraceutical (or functional food) products that can assist to regulate blood glucose levels in patients with type 2 diabetes [11, 12]. Animal studies in diabetic and non-diabetic rats have suggested that certain individual flavonoids and flavonoid-containing seed extracts have a hypoglycemic effect due to their effect on glycogen metabolism [13-15]. The inhibitory potency of common natural flavonoids on the activity of GP has been examined and showed that certain flavonoids are potent inhibitors of the enzyme [16]. However, the molecular basis of their inhibitory potency has not yet been established. Flavopiridol (Table 1), a synthetic flavonoid has been shown to potently inhibit GP in vitro $\left(K_{\mathrm{i}}=1.2 \mu \mathrm{M}\right)$ and ex vivo [17] by binding to the inhibitor site of the enzyme [18]. Likewise, four olefin derivatives of flavopiridol are some of the most potent inhibitors of $\mathrm{GPb}$ in vitro $\left(K_{\mathrm{i}}=1-1.9 \mu \mathrm{M}\right)$ and ex vivo and they also bind to the inhibitor site [17]. Chrysin (Table 1), a natural occurring flavonoid, is a potent inhibitor $\left(K_{\mathrm{i}}=19 \mu \mathrm{M}\right)$ that also binds to the inhibitor site [19], while another flavonoid inhibitor, quercetagetin, binds to the allosteric site [20]. A recent screening of thirteen polyphenolic extracts obtained from the vinification byproducts of Vitis vinifera against GP revealed that the most active ingredient of these extracts is quercetin which binds to a novel binding site, distinct from the other known sites of the enzyme [7]. One of the most potent natural flavonoid inhibitors of GP is ellagic acid with an $I C_{50}$ value of $3.2 \mu \mathrm{M}$ [20]. However, currently no information about the binding mode of this ligand exists which would facilitate structure-based inhibitor 
design and optimization efforts. Since natural flavonoids are potent inhibitors of GP that display potency also at the cellular level it is of significant interest to elucidate the molecular details that govern their inhibitory potency. Thus, we have studied in detail the binding of gallic acid and its dimer ellagic acid (both natural compounds) to GP by kinetics and X-ray crystallography methods.

\section{Materials and Methods}

Ellagic and gallic acid were purchased from Sigma-Aldrich. Rabbit skeletal muscle glycogen phosphorylase $b$ $(\mathrm{GPb})$ was isolated, and purified as described previously [21]. GPa was prepared from GPb by phosphorylation with a truncated form of the $\gamma$ (catalytic) subunit of rabbit skeletal muscle phosphorylase kinase produced as described previously [22]. Kinetic studies were performed at $30^{\circ} \mathrm{C}$ in the direction of glycogen synthesis by measuring the inorganic phosphate released in the reaction [21]. Briefly, the enzyme $(5 \mathrm{\mu g} / \mathrm{ml}$ ) was assayed in a $30 \mathrm{mM}$ imidazole/ $\mathrm{HCl}$ buffer ( $\mathrm{pH} \mathrm{6.8)} \mathrm{containing} 60 \mathrm{mM} \mathrm{KCl}, 0.6 \mathrm{mM}$ EDTA, and $0.6 \mathrm{mM}$ dithiothreitol using constant concentrations of glycogen $(0.2 \% \mathrm{w} / \mathrm{v}), \mathrm{AMP}(1 \mathrm{mM})$, and various concentrations of Glc-1-P and inhibitors. Initial velocities were calculated from the pseudo-first order rate constants. Kinetic data presented in the form of a Lineweaver-Burk, Dixon or Hill plots were analysed for the calculation of the kinetic and binding parameters by the non-linear regression program GRAFIT [23]. $\mathrm{GPb}$ crystals were grown in the tetragonal lattice, space group $P 4_{3} 2_{1} 2$, as described previously [24]. X-ray crystallography studies were performed by diffusion of a $100 \mathrm{mM}$ solution of gallic acid or $10 \mathrm{mM}$ of ellagic acid in the crystallization media (supplemented with $15 \%(v / v)$ DMSO in the case of ellagic acid) in preformed GPb crystals at room temperature for 12 hours prior to data collection. X-ray diffraction data were collected using synchrotron radiation for the GPb - ellagic acid complex on station ID911-2 at MAXLab Synchrotron Radiation Source in Lund, Sweden. For the GPb - gallic acid complex data were collected on station P14 of the EMBL - Hamburg outstation. Crystal orientation, integration of reflections, interframe scaling, partial reflection summation, and data reduction was performed by the program Mosflm [25] for the GPb - ellagic acid complex and XDS [26] for the GPb - gallic acid complex. Scaling and merging of intensities were performed by SCALA [27]. Crystallographic refinement of the complexes was performed by maximum-likelihood methods using REFMAC [27]. The starting model employed for the refinement of the complexes was the structure of the native $\mathrm{T}$ state GPb complex determined at $1.9 \AA$ resolution (Leonidas et al., unpublished results). Ligand molecule coordinates and topologies were obtained from the REFMAC library and they were fitted to the electron density maps after adjustment of their torsion angles. Alternate cycles of manual rebuilding with the molecular graphic program COOT [27] and refinement with REFMAC [28] improved the quality of the models. A summary of the data processing and refinement statistics for the inhibitor complex structures is given in Table 2. The stereochemistry of the protein residues was validated by MolProbity [29]. Hydrogen bonds and van der Waals interactions were calculated with the program CONTACT as implemented in CCP4 [27] applying a distance cut off $3.3 \AA$ and $4.0 \AA$, respectively. Figures were prepared with PyMol [30]. The coordinates of the new structures have been deposited with the RCSB Protein Data Bank (http://www.rcsb.org/pdb) with codes presented in Table 2.

\section{Results and Discussion}

\section{Determination of inhibition constants $\left(K_{i}\right)$}

Although the target enzyme is the human liver GP, our studies were performed with the rabbit muscle isoform. However, the inhibitor site is identically conserved in all mammalian GPs [31], indicating that compounds inhibiting muscle GP by binding at this site are likely to inhibit also liver GP. Both gallic and ellagic acid are competitive inhibitors with respect to Glc-1-P as revealed by the Lineweaver-Burk plots that 
intersect at the same point on the $y$-axis for GPb and GPa. The $K_{\mathrm{i}}$ values derived from the Dixon plots (1/v vs [I]) were for gallic acid $1.73 \pm 0.16 \mathrm{mM}$ and $3.86 \pm 0.27 \mathrm{mM}$ for GPb and GPa, respectively. For ellagic acid they were found to be $13.4 \pm 1.2 \mu \mathrm{M}$ and $7.52 \pm 0.36 \mu \mathrm{M}$ for GPb and GPa, respectively. Inhibition constants for ellagic acid are an order of magnitude lower than those of gallic acid. Although the $K_{\mathrm{i}}$ value for ellagic acid is similar to that of chrysin $\left(K_{\mathrm{i}}=19 \mu \mathrm{M}\right)$ [19], it remains $\sim 10$ fold higher than that of flavopiridol $\left(K_{\mathrm{i}}=1.2 \mu \mathrm{M}\right)$ [19]. Gallic acid appears to be a poor inhibitor of both enzyme forms since its $K_{\mathrm{i}}$ values are significantly higher than those of caffeine $(0.13 \mathrm{mM}$ and $0.17 \mathrm{mM}$ for GPb and GPa, respectively [32]). These $K_{\mathrm{i}}$ values for ellagic acid are in good agreement with the $I C_{50}$ values measured in a previous study [16] (12.1 $\mu \mathrm{M}$ and 3.2 $\mu \mathrm{M}$ for GPb and GPa, respectively). Furthermore, in agreement with our studies which show that gallic acid displays $K_{\mathrm{i}}$ values in the range of $\mathrm{mM}$ in that study [16] gallic acid did not show any inhibition of both enzyme forms in concentrations up to $50 \mu \mathrm{M}$.

\section{Multiple Inhibition Studies}

Caffeine and AMP are two GP ligands that bind to the inhibitor and to the allosteric sites of GP, respectively [1]. To establish whether gallic and ellagic acids interact with the allosteric or the inhibitor binding site, the inhibition behaviour of both compounds was investigated with respect to caffeine and AMP. The kinetic data were analysed by Dixon plots from which the interaction constant $(\alpha)$ between multiple inhibitors can be estimated [33]. Thus, with varying concentrations of caffeine and ellagic or gallic acids, and constant concentrations of AMP (1 mM) and substrate (Glc-1-P; $20 \mathrm{mM}$ ) the Dixon plots for both GPb (Fig. 1) and $\mathrm{GPa}$, yielded a pattern of parallel lines (interaction constant approaches infinity), indicating that the binding of ellagic or gallic acid and caffeine is mutually exclusive (they compete for the same binding site) [33]. Similar analysis with varying concentrations of AMP and constant concentrations of substrate (GIc-1-P; 2.5 $\mathrm{mM}$ ) yielded intersected lines in the Dixon plots for both GPb (Fig. 2) and GPa, signifying that gallic or ellagic acids and AMP are not mutually exclusive (they can both bind at the enzyme) [33]. These experiments indicated that gallic and ellagic acids bind to the inhibitor binding site.

Caffeine functions with glucose (the physiological inhibitor of the enzyme) in a strongly synergistic mode [34] with an interaction constant $\alpha=0.2$. Thus, we investigated whether the binding of gallic or ellagic acid to GPb interacts with that of glucose. We carried out initial velocity studies by varying gallic acid, ellagic acid, and glucose concentrations at fixed concentrations of the substrates Glc-1-P (2.5 mM) and glycogen (0.2\%). The results are shown in Fig. 3 . The degree of synergism with glucose differs significantly between the two compounds. The binding of gallic acid does not interact with glucose binding $(\alpha=1.0)$, while the binding of ellagic acid is strongly synergistic with that of glucose $(\alpha=0.22)$. Thus, only inhibition of ellagic acid is synergistic with glucose. This means that ellagic acid and glucose are able to bind to the enzyme at the same time [33], and each compound promotes the binding of the other, like caffeine and glucose [34]. The effect of glucose on the potency of ellagic acid could be an important physiological feature for a liver GPa inhibitor, because the decrease in inhibitor potency as glucose concentrations decrease in vivo should diminish the risk of hypoglycemia. This has been also suggested for another pair of a potent inhibitor and glucose [35].

\section{Structural studies}

In order to provide a structural explanation for the gallic and ellagic acids inhibition of GPb, we have determined the crystal structures of the GPb-gallic acid and the GPb-ellagic acid complexes by X-ray crystallography at 2.1 and $2.0 \AA$ resolution, respectively. Both inhibitors were found bound to the inhibitor site, in agreement with the kinetic experiments, and all ligand atoms are well defined within the electron 
density maps (Fig. 4). There are not any significant conformational changes of the polypeptide chain on binding gallic acid or ellagic acid. Superposition of the native T state GPb structure with the GPb-gallic acid and the GPb-ellagic acid complex structures over well-defined residues gave a r.m.s.d. of 0.21 and $0.17 \AA$ for $\mathrm{Ca}$ atoms for the gallic and ellagic acids protein complexes, respectively. The binding modes of gallic acid and ellagic acid are similar. The most characteristic feature is the stacking interactions of the inhibitors with the aromatic side chains of Phe285 (from the 280s loop) and Tyr613 (Fig. 5a). On binding at the inhibitor site gallic acid forms a single hydrogen bond interaction with Asn282 side chain atoms and it is involved in water mediated hydrogen bond interactions with atoms from residues Asp283, Ile570, Ala 610, and Gly612 (Table 3). Gallic acid and GPb residues are involved in 58 van der Waals interactions that mainly involve atoms from Phe285 and Tyr613. The plane of the gallic acid ring makes an angle of about $10^{\circ}$ with the Tyr613 aromatic plane lying at distances varied between 3.7 and $4.3 \AA$, while it is almost parallel to the plane of the benzyl ring of Phe285, at a distance of $3.7 \AA$ (Fig. 5A). Ellagic acid on binding to the inhibitor binding site forms a single strong hydrogen bond to the side chain atom ND of Asn282 and it is involved in water-mediated hydrogen bond interactions with atoms from Asp283, Ile570, Ala610, Gly612, His614, and Met615 (Table 4). The plane of the ellagic acid lies at distances 3.4 and $3.6 \AA$ from the planes of benzyl ring of Phe285 and Try613, respectively. The ellagic acid plane is parallel to the plane of the Phe285 phenyl ring and at an angle of $24^{\circ}$ from the plane of the hydroxyphenyl ring of Tyr613 (Fig. 5B). Due to its larger size, the ellagic acid exploits almost twice the number of van der Waals interactions with GPb upon binding (95) than the gallic acid (58). On forming the inhibitor complexes both ligands become buried. The solvent accessibilities of the free and bound ligand molecules are 299 and $78 \AA^{2}$ (gallic acid), and 427 and $116 \AA^{2}$ (ellagic acid), respectively, indicating that $\sim 74 \%$ of the inhibitor surface becomes buried. This comes almost entirely from the non-polar groups. On the protein surface, a total of 669 and $772 \AA^{2}$ solvent accessible surface area becomes inaccessible on binding of the gallic and the ellagic acids, respectively. The total buried surface areas (protein plus ligand) are $371 \AA^{2}$ and $532 \AA^{2}$ for the gallic and ellagic acids GPb complexes. The corresponding values in the GPb-caffeine [36], GPb-flavopiridol [36], GPb-CP320626 [37] and GPb-W1807 [38] complexes are $467 \AA^{2}, 618 \AA^{2}, 819 \AA^{2}$ and $852 \AA^{2}$, respectively. These areas show an approximate correlation with the ligand affinity since they follow the order of potency of the ligands: gallic acid $\left(K_{\mathrm{i}}=1.7 \mathrm{mM}\right)$, caffeine $\left(K_{\mathrm{i}}=0.13 \mathrm{mM}\right)$ [32], ellagic acid $\left(K_{\mathrm{i}}=13.4 \mu \mathrm{M}\right)$, flavopiridol $\left(K_{\mathrm{i}}=1.2 \mu \mathrm{M}\right)$ [19] CP320626 (/ $\left.C_{50}=334 \mathrm{nM}\right)[37]$ and $\mathrm{W} 1807\left(K_{\mathrm{i}}=1.6 \mathrm{nM}\right)[38]$.

\section{Comparison with other flavonoids}

The most potent flavonoid inhibitors that bind to the GPb inhibitor site, reported thus far, are flavopiridol $\left(K_{\mathrm{i}}=1.2 \mu \mathrm{M}\right)$ and chrysin $\left(K_{\mathrm{i}}=19 \mu \mathrm{M}\right)$ [19] (Table 1). Although the structural mode of their binding is very similar, the source of the greater inhibition of flavopiridol was attributed by QM/MM-PBSA calculations to favourable interactions of its 4-hydroxypiperidin-1-yl group with GPb without significant desolvation costs [19]. Ellagic acid displays a better inhibitory potency than chrysin. Comparative structural analysis of the binding of ellagic acid and flavopiridol to GPb reveals that although both ligands bind between the benzyl rings of Phe285 and Tyr613 there are significant differences. Thus, flavopiridol stacks ring A (Table 1) against the phenyl ring of Phe285 and ring $C$ against the hydroxyl-phenyl ring of Tyr613. Ellagic acid has its rings B and A co-planar against the aromatic side chains of Phe285 and Tyr613, respectively. Thus, flavopiridol is placed deeper in the inhibitor site cleft than ellagic acid (Fig. 6). Moreover, the planes formed by the aromatic rings of flavopiridol and ellagic acid are inclined to each other by $\sim 15 \%$ (Fig. 6). Flavopiridol being bulkier than ellagic acid exploits 12 additional van der Waals interactions with the protein. In contrast 
to ellagic acid which forms a strong hydrogen bond interaction with Asn282, flavopiridol does not participate in any direct hydrogen bond with protein residues. Both ligands are involved in water-mediated hydrogen bond interactions with GPb. Therefore, it seems that the difference in the potency between flavopiridol and ellagic acid might be attributed to the interactions of the 4-hydroxypiperidin-1-yl group of flavopiridol with GPb, like in the case of chrysin and flavopiridol [19].

Flavonoids are natural abundant compounds found ubiquitously in plants and the most common group of polyphenolic compounds in the human diet. The present study establishes ellagic acid as the most potent natural compound that binds to the GP inhibitor site. The inhibitory potency of ellagic acid is even stronger for GPa than for GPb, and since GPa is the physiologically active form of the enzyme this fact adds more value to a potential antihyperglycaemic usage of ellagic acid. Furthermore, our results can serve as the starting point for the exploitation of food products that contain ellagic acid for antidiabetic usage and the development of novel biofunctional food and dietary supplements/herbal medicines, an increasingly important approach to both diabetes treatment and prevention.

\section{Acknowledgments}

This project was implemented under the "ARISTEIA" Action of the "Operational Programme Education and Lifelong Learning" and is co-funded by the European Social Fund (ESF) and National Resources. This work was supported in part by the Postgraduate Programmes "Biotechnology-Quality assessment in Nutrition and the Environment", "Application of Molecular Biology-Molecular Genetics-Molecular Markers", Department of Biochemistry and Biotechnology, University of Thessaly. Work at the Synchrotron Radiation Sources, MAX-lab, Lund, Sweden and EMBL Hamburg Outstation, Germany, was supported from the EU FP7 Programme (FP7/2007-2013) under BioStruct-X (grant agreement N²83570).

\section{References}

1. Oikonomakos, N. G. (2002) Glycogen phosphorylase as a molecular target for type 2 diabetes therapy, Curr Protein Pept Sci. 3, 561-86.

2. Somsak, L., Czifrak, K., Toth, M., Bokor, E., Chrysina, E. D., Alexacou, K. M., Hayes, J. M., Tiraidis, C., Lazoura, E., Leonidas, D. D., Zographos, S. E. \& Oikonomakos, N. G. (2008) New inhibitors of glycogen phosphorylase as potential antidiabetic agents, Curr Med Chem. 15, 2933-83.

3. Oikonomakos, N. G. \& Somsak, L. (2008) Advances in glycogen phosphorylase inhibitor design, Curr Opin Investig Drugs. 9, 379-395.

4. Hayes, J. M., Kantsadi, A. L. \& Leonidas, D. D. (2014) Natural products and their derivatives as inhibitors of glycogen phosphorylase: potential treatment for type 2 diabetes, Phytochemistry Reviews. 13, 471-498.

5. Agius, L. (2010) Physiological control of liver glycogen metabolism: lessons from novel glycogen phosphorylase inhibitors, Mini Rev Med Chem. 10, 1175-87.

6. Monod, J., Wyman, J. \& Changeux, J. P. (1965) On the Nature of Allosteric Transitions: A Plausible Model, J Mol Biol. 12, 88-118.

7. Kantsadi, A. L., Apostolou, A., Theofanous, S., Stravodimos, G. A., Kyriakis, E., Gorgogietas, V. A., Chatzileontiadou, D. S., Pegiou, K., Skamnaki, V. T., Stagos, D., Kouretas, D., Psarra, A. M., Haroutounian, S. A. \& Leonidas, D. D. (2014) Biochemical and biological assessment of the inhibitory potency of extracts from vinification byproducts of Vitis vinifera extracts against glycogen phosphorylase, Food Chem Toxicol. 67, 3543.

8. Somsak, L. (2011) Glucose derived inhibitors of glycogen phosphorylase, Comptes Rendus Chimie. 14, 211-223.

9. Kasvinsky, P. J., Fletterick, R. J. \& Madsen, N. B. (1981) Regulation of the dephosphorylation of glycogen phosphorylase a and synthase b by glucose and caffeine in isolated hepatocytes, Can J Biochem. 59, 387-95. 10. Ercan-Fang, N. \& Nuttall, F. Q. (1997) The effect of caffeine and caffeine analogs on rat liver phosphorylase a activity, J Pharmacol Exp Ther. 280, 1312-8. 
11. Kalra, E. K. (2003) Nutraceutical--definition and introduction, AAPS PharmSci. 5, E25.

12. Whitman, M. (2001) Understanding the perceived need for complementary and alternative nutraceuticals: lifestyle issues, Clin J Oncol Nurs. 5, 190-4.

13. Ong, K. C. \& Khoo, H. E. (2000) Effects of myricetin on glycemia and glycogen metabolism in diabetic rats, Life Sci. 67, 1695-705.

14. Ahmad, M., Akhtar, M. S., Malik, T. \& Gilani, A. H. (2000) Hypoglycaemic action of the flavonoid fraction of Cuminum nigrum seeds, Phytother Res. 14, 103-6.

15. Anila, L. \& Vijayalakshmi, N. R. (2000) Beneficial effects of flavonoids from Sesamum indicum, Emblica officinalis and Momordica charantia, Phytother Res. 14, 592-5.

16. Jakobs, S., Fridrich, D., Hofem, S., Pahlke, G. \& Eisenbrand, G. (2006) Natural flavonoids are potent inhibitors of glycogen phosphorylase, Mol Nutr Food Res. 50, 52-7.

17. Hampson, L. J., Arden, C., Agius, L., Ganotidis, M., Kosmopoulou, M. N., Tiraidis, C., Elemes, Y., Sakarellos, C., Leonidas, D. D. \& Oikonomakos, N. G. (2006) Bioactivity of glycogen phosphorylase inhibitors that bind to the purine nucleoside site, Bioorg Med Chem. 14, 7835-45.

18. Oikonomakos, N. G., Schnier, J. B., Zographos, S. E., Skamnaki, V. T., Tsitsanou, K. E. \& Johnson, L. N. (2000) Flavopiridol inhibits glycogen phosphorylase by binding at the inhibitor site., J Biol Chem. 275, 34566-34573.

19. Tsitsanou, K. E., Hayes, J. M., Keramioti, M., Mamais, M., Oikonomakos, N. G., Kato, A., Leonidas, D. D. \& Zographos, S. E. (2013) Sourcing the affinity of flavonoids for the glycogen phosphorylase inhibitor site via crystallography, kinetics and QM/MM-PBSA binding studies: Comparison of chrysin and flavopiridol, Food Chem Toxicol. 61, 14-27.

20. Kato, A., Nasu, N., Takebayashi, K., Adachi, I., Minami, Y., Sanae, F., Asano, N., Watson, A. A. \& Nash, R. J. (2008) Structure-activity relationships of flavonoids as potential inhibitors of glycogen phosphorylase, $J$ Agric Food Chem. 56, 4469-4473.

21. Tsirkone, V. G., Tsoukala, E., Lamprakis, C., Manta, S., Hayes, J. M., Skamnaki, V. T., Drakou, C., Zographos, S. E., Komiotis, D. \& Leonidas, D. D. (2010) 1-(3-Deoxy-3-fluoro-beta-d-glucopyranosyl) pyrimidine derivatives as inhibitors of glycogen phosphorylase b: Kinetic, crystallographic and modelling studies, Bioorg Med Chem. 18, 3413-25.

22. Hayes, J. M., Skamnaki, V. T., Archontis, G., Lamprakis, C., Sarrou, J., Bischler, N., Skaltsounis, A. L., Zographos, S. E. \& Oikonomakos, N. G. (2011) Kinetics, in silico docking, molecular dynamics, and MM-GBSA binding studies on prototype indirubins, KT5720, and staurosporine as phosphorylase kinase ATP-binding site inhibitors: the role of water molecules examined, Proteins. 79, 703-19.

23. Leatherbarrow, R. J. (1998) GraFit version 4.06, Erithacus Software Ltd Staines, UK.

24. Alexacou, K. M., Tenchiu Deleanu, A. C., Chrysina, E. D., Charavgi, M. D., Kostas, I. D., Zographos, S. E., Oikonomakos, N. G. \& Leonidas, D. D. (2010) The binding of beta-d-glucopyranosyl-thiosemicarbazone derivatives to glycogen phosphorylase: A new class of inhibitors, Bioorg Med Chem. 18, 7911-22.

25. Leslie, A. G. W. \& Powell, H. R. (2007) Processing Diffraction Data with Mosflm in Evolving Methods for Macromolecular Crystallography (Read, R. J. \& Sussman, J. I., eds) pp. 41-51, Springer.

26. Kabsch, W. (2010) Xds, Acta Crystallogr D Biol Crystallogr. 66, 125-32.

27. CCP4 (1994) The CCP4 suite : programs for protein crystallography, Acta Crystallogr. D 50, 760-763.

28. Murshudov, G. N., Skubak, P., Lebedev, A. A., Pannu, N. S., Steiner, R. A., Nicholls, R. A., Winn, M. D., Long, F. \& Vagin, A. A. (2011) REFMAC5 for the refinement of macromolecular crystal structures, Acta Crystallogr D Biol Crystallogr. 67, 355-67.

29. Chen, V. B., Arendall, W. B., 3rd, Headd, J. J., Keedy, D. A., Immormino, R. M., Kapral, G. J., Murray, L. W., Richardson, J. S. \& Richardson, D. C. (2010) MolProbity: all-atom structure validation for macromolecular crystallography, Acta Crystallogr D Biol Crystallogr. 66, 12-21.

30. DeLano, W. L. (2002) The PyMol Molecular Visualization System in Sa Carlos, CA, USA.

31. Hudson, J. W., Golding, G. B. \& Crerar, M. M. (1993) Evolution of allosteric control in glycogen phosphorylase, J Mol Biol. 234, 700-21.

32. Papageorgiou, A. C., Oikonomakos, N. G. \& Leonidas, D. D. (1989) Inhibition of rabbit muscle glycogen phosphorylase by D- gluconohydroximo-1,5-lactone-N-phenylurethane, Arch Biochem Biophys. 272, 376385.

33. Segel, I. H. (1993) Enzyme Kinetics, Wiley Interscience, New York. 
34. Madsen, N. B., Shechosky, S. \& Fletterick, R. J. (1983) Site-site interactions in glycogen phosphorylase b probed by ligands specific for each site, Biochemistry. 22, 4460-5.

35. Martin, W. H., Hoover, D. J., Armento, S. J., Stock, I. A., McPherson, R. K., Danley, D. E., Stevenson, R. W., Barrett, E. J. \& Treadway, J. L. (1998) Discovery of a human liver glycogen phosphorylase inhibitor that lowers blood glucose in vivo, Proc Natl Acad Sci USA. 95, 1776-1781.

36. Oikonomakos, N. G., Schnier, J. B., Zographos, S. E., Skamnaki, V. T., Tsitsanou, K. E. \& Johnson, L. N. (2000) Flavopiridol inhibits glycogen phosphorylase by binding at the inhibitor site, J Biol Chem. 275, 3456673.

37. Oikonomakos, N. G., Skamnaki, V. T., Tsitsanou, K. E., Gavalas, N. G. \& Johnson, L. N. (2000) A new allosteric site in glycogen phosphorylase b as a target for drug interactions., Structure. 8, 575-584.

38. Zographos, S. E., Oikonomakos, N. G., Tsitsanou, K. E., Leonidas, D. D., Chrysina, E. D., Skamnaki, V. T., Bischoff, H., Goldmann, S., Watson, K. A. \& Johnson, L. N. (1997) The structure of glycogen phosphorylase b with an alkyldihydropyridine-dicarboxylic acid compound, a novel and potent inhibitor, Structure. 5, 141325. 


\section{Tables}

Table 1. The chemical structures of inhibitors known to bind at the GP inhibitor site together with their $K_{i}^{\prime} \mathrm{s}$ for $\mathrm{GPb}$ inhibition.

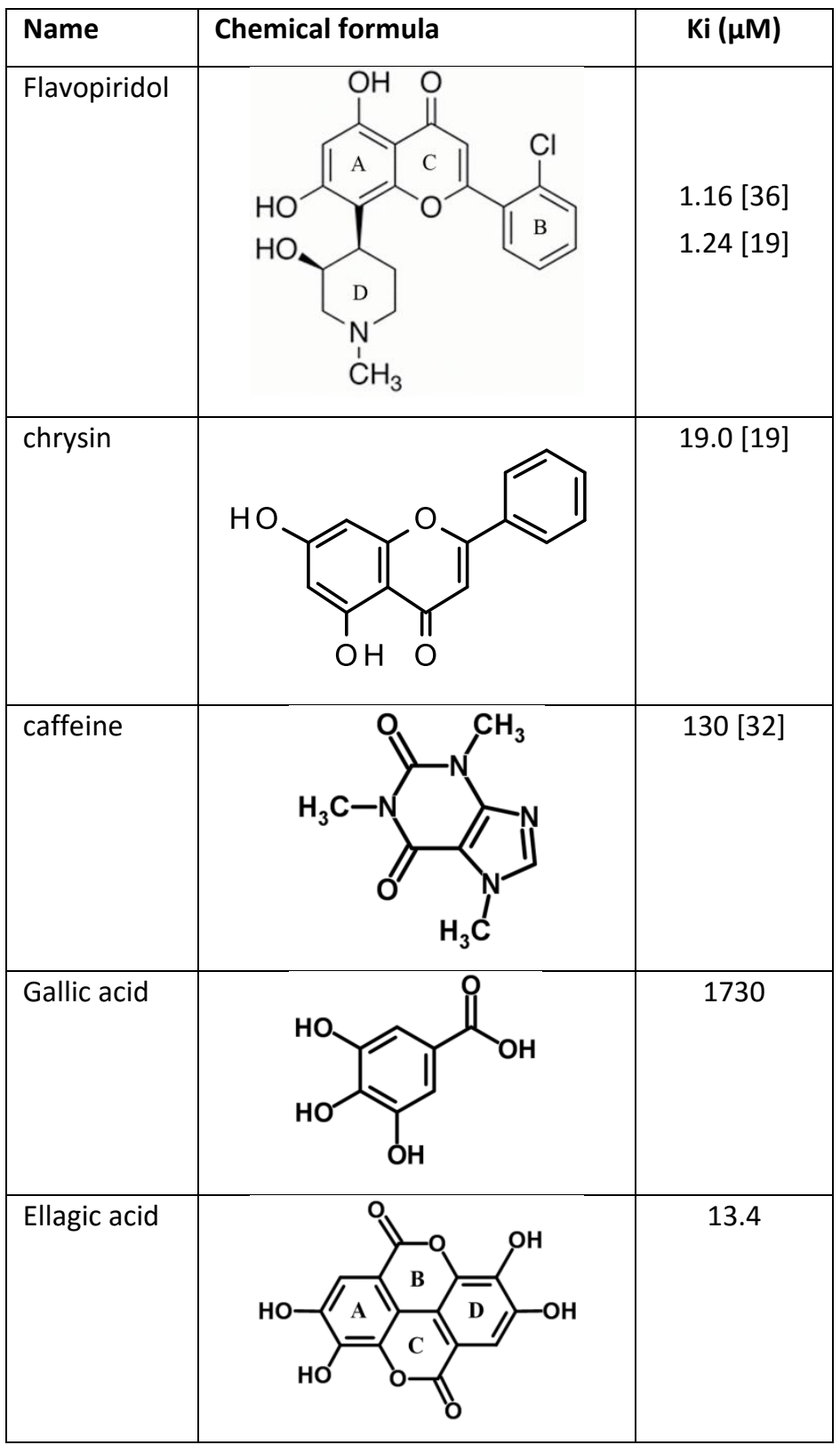

Table 2: Summary of the diffraction data processing and refinement statistics for the GPb complexes

\begin{tabular}{|l|c|c|}
\hline GPb complex & Ellagic acid & Gallic Acid \\
\hline \multicolumn{2}{|l|}{ Data collection and processing statistics } \\
\hline Resolution $(\AA)$ & $38.35-2.00$ & $86.36-2.10$ \\
\hline Outermost shell (Å) & $2.11-2.00$ & $2.21-2.10$ \\
\hline Reflections measured & $217233(31425)$ & $375790(55894)$ \\
\hline Unique reflections ${ }^{[a]}$ & $63707(9384)$ & $56323(8072)$ \\
\hline Multiplicity ${ }^{[a]}$ & $3.4(3.3)$ & $6.7(6.9)$ \\
\hline
\end{tabular}




\begin{tabular}{|c|c|c|}
\hline$R_{\text {symm }}^{[\mathrm{a}]}$ & $0.100(0.430)$ & $0.077(0.497)$ \\
\hline Completeness (\%) & $97.2(98.8)$ & $98.4(97.8)$ \\
\hline$\langle l / \sigma \mid\rangle^{[a]}$ & $5.6(2.7)$ & $15.4(5.1)$ \\
\hline \multicolumn{3}{|l|}{ Refinement statistics } \\
\hline$R_{\text {cryst }}^{\text {[a] }}$ & $0.173(0.251)$ & $0.159(0.215)$ \\
\hline$R_{\text {free }}{ }^{[a]}$ & $0.202(0.291)$ & $0.189(0.219)$ \\
\hline No of solvent molecules & 364 & 221 \\
\hline \multicolumn{3}{|c|}{ r.m.s. deviation from ideality } \\
\hline in bond lengths $(\AA)$ & 0.009 & 0.009 \\
\hline in angles $\left({ }^{\circ}\right)$ & 1.3 & 1.4 \\
\hline \multicolumn{3}{|l|}{ Average $B$ factor $\left(\AA^{2}\right)$} \\
\hline Protein atoms & 29.8 & 42.3 \\
\hline Solvent molecules & 34.3 & 43.1 \\
\hline Inhibitor atoms & 61.2 & 56.8 \\
\hline PDB entry & 4YUA & $4 Z 5 X$ \\
\hline
\end{tabular}

Table 3: Gallic acid interactions with Glycogen phosphorylase b residues in the crystal.

\begin{tabular}{|l|l|l|}
\hline \multirow{2}{*}{$\begin{array}{c}\text { Inhibitor } \\
\text { atom }\end{array}$} & \multicolumn{2}{|c|}{ Protein residues (atoms) } \\
\cline { 2 - 3 } CAH & Hydrogen bonds (distance in $\boldsymbol{A}$ ) & Van der Waals interactions \\
\hline OAA & & Tyr613 (CD1), Phe285 (CE1) \\
\hline CAK & & Tyr613 (CE1) \\
\hline CAF & & Tyr613 (CD1, CG), Phe285 (CD1, CE1, CZ) \\
\hline CAG & & Tyr613 (CD1, CE1, CZ, CE2, CD2, CG), Phe285 (CG, CD1, CE1) \\
\hline CAJ & & Tyr613 (CB, CG), Phe285 (CD2, CZ, CE2) \\
\hline OAD & Water-184 (3.3) & Tyr613 (CB), Phe285 (CG, CD2, CE2) \\
\hline CAL & & Ala610 (CB), Phe285 (CD2), Asn282 (OD1), \\
\hline OAE & Asn282 OD1 (3.3), Water-31 (2.4) & Asn282 (CA, CB, CG, OD1), Ala610 (CB), Phe285 (CB, CG, CD2) \\
\hline CAI & \multicolumn{1}{|c|}{$\mathbf{4}$} & Tyr613 (CE2, CD2, CG), Phe285 (CB, CG, CD1, CD2), Water-31 (O) \\
\hline OAC & Water-31 (2.7) & His571 (NE2, CD2), Asp283 (O), Tyr613 (CE2, CD2), Phe285 (CB, CG) \\
\hline Total & & $\mathbf{5 8}$ \\
\hline
\end{tabular}

Table 4: Ellagic acid interactions with glycogen phosphorylase b residues in the crystal.

\begin{tabular}{|l|l|l|}
\hline \multirow{2}{*}{$\begin{array}{c}\text { Inhibitor } \\
\text { atom }\end{array}$} & \multicolumn{2}{|c|}{ Protein residues (atoms) } \\
\cline { 2 - 3 } & Hydrogen bonds(distance in $\boldsymbol{A}$ ) & Van der Waals interactions \\
\hline C1 & & Gly612 (C, O), Phe285 (CZ, CE2) \\
\hline C2 & & Tyr613 (CB), Phe285 (CG, CD2, CE1, CZ, CE2) \\
\hline C3 & & Tyr613 (CG, CD1), Phe285 (CG, CD1, CD2, CE1, CZ, CE2) \\
\hline C4 & Pater-227 (3.3) & Phe285 (CE1, CZ) \\
\hline O5 & & Phe285 (CZ) \\
\hline C6 & & Phe285 (CZ), Gly612 (O), Water-227 (O) \\
\hline C7 & Tyr613 (CG, CD1, CE2, CD2), Phe285 (CB, CG, CD1, CE1) \\
\hline
\end{tabular}




\begin{tabular}{|l|l|l|}
\hline C8 & & Tyr613 (CD1, CE1, CZ, OH, CE2, CD2), His571:A (NE2), Phe285 (CD1) \\
\hline C9 & & Tyr613 (CE1, CZ, OH) \\
\hline C10 & & Tyr613 (CE1), Phe285 (CE1) \\
\hline C11 & Water-33 (3.3) & Ala610 (CB), Tyr613 (CB), Phe285 (CG, CD2, CE2) \\
\hline O12 & & Ala610 (CB), Phe285 (CB, CG, CD2) \\
\hline C13 & & Tyr613 (CG, CE2, CD2), Phe285 (CB, CG, CD1), Water-33 (O) \\
\hline C14 & & Gly612 (CA, C, O), Phe285 (CE2), \\
\hline C15 & & Gly612 (CA, C) \\
\hline C16 & & Ala610 (CB), Phe285 (CD2), Asn282:A (ND2) \\
\hline O20 & & $\begin{array}{l}\text { Tyr613 (CE1, CZ, OH), Glu382 (OE2), His571:A (CE1, NE2), Glu572 } \\
\text { (OE2) }\end{array}$ \\
\hline O21 & Water-33 (2.8) & $\begin{array}{l}\text { His571:B (CE1), His571:A (CD2, NE2), Asp283 (O), Tyr613 (CE2, } \\
\text { CD2), Phe285 (CB, CG) }\end{array}$ \\
\hline O22 & Asn282 ND (2.2) & Ala610 (CB), Asn282:A (CB, CG), Asn282:B (CB, ND2) \\
\hline O23 & & Gly612 (CA, N), Asn282:A (ND2), Glu287 (OE2) \\
\hline O24 & Water-227 (3.1) & Gly612 (O) \\
\hline Total & $\mathbf{5}$ & \\
\hline
\end{tabular}



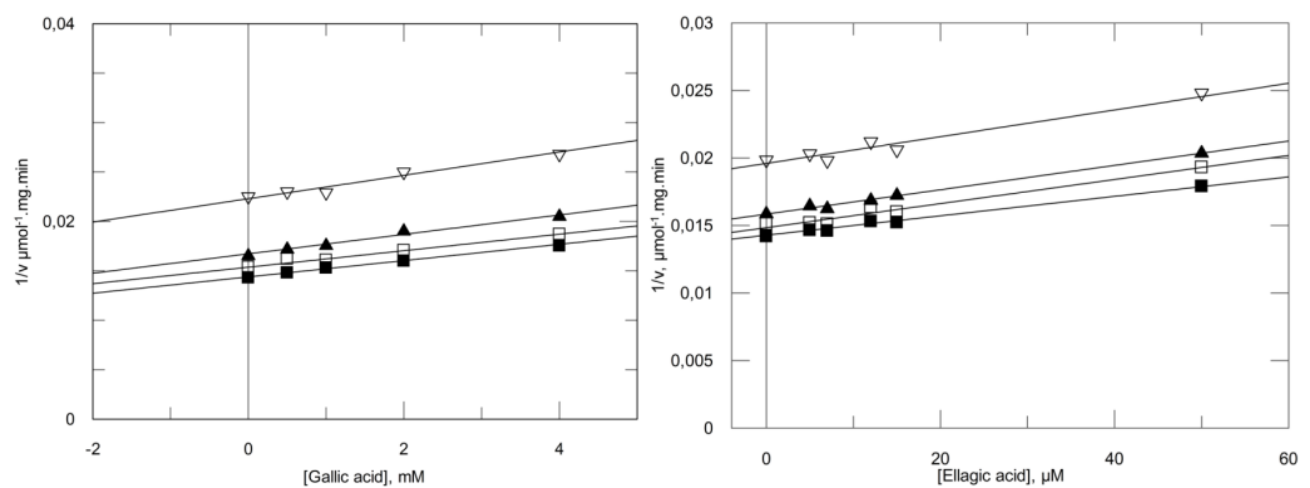

Figure 1. Competition of gallic (A) or ellagic acid (B) inhibition and caffeine. Dixon plots for $\mathrm{GPb}$ at constant concentrations of Glc-1-P (20 mM), AMP (1.0 mM) and glycogen $(0.2 \%)$ and various concentrations of inhibitors. Caffeine concentrations were as follows: $0.1 \mathrm{mM}(\boldsymbol{\square}), 0.2 \mathrm{mM}(\square), 0.4 \mathrm{mM}(\mathbf{\Delta})$ and $0.8 \mathrm{mM}(\nabla)$.
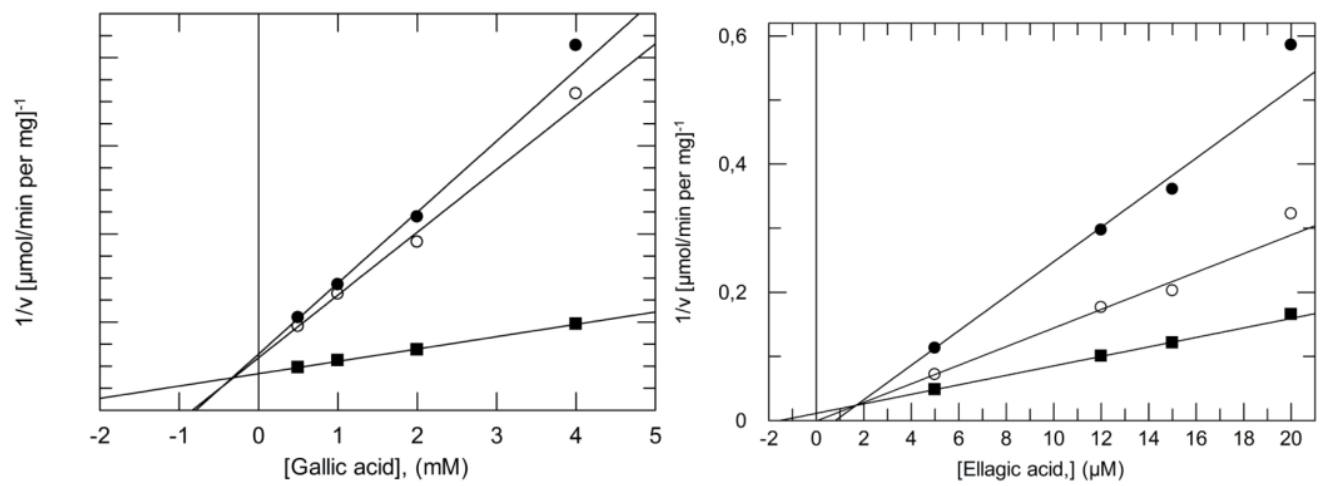

Figure 2. The lack of competition between gallic (A) or ellagic acid (B) and AMP. Inhibition of GPb was measured in at constant concentrations of Glc-1-P (2.5 mM), and glycogen $(0.2 \%)$ and various concentrations of AMP. AMP concentrations were as follows: $0.1 \mathrm{mM}(\mathbf{O}), 0.2 \mathrm{mM}(\mathrm{O})$, and $1.0 \mathrm{mM}(\mathbf{\square})$ in the Dixon plot for gallic acid (A) and $50 \mu \mathrm{M}(\mathbf{O}), 100 \mu \mathrm{M}(\mathrm{O})$ and $200 \mu \mathrm{M}(\mathbf{\square})$ for the plot of ellagic acid (B).
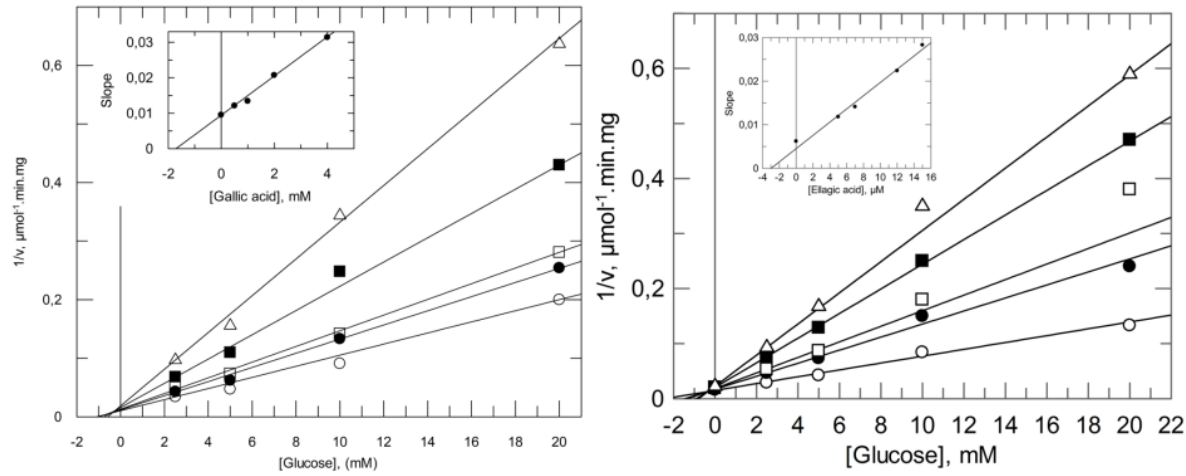

Figure 3. Inhibition of GPb by mixtures of gallic (A) or ellagic (B) acid and glucose. (A) Dixon plot of reciprocal velocity versus glucose concentrations at different gallic acid concentrations and constant concentrations of Glc-1-P (20 mM), AMP (1 mM), and glycogen (0.2\%). Gallic acid concentrations were 0 $(\mathrm{O}), 0.5(\mathbf{O}), 1(\square), 2(\square)$, and $4 \mathrm{mM}(\Delta)$. The replot of the slopes versus gallic acid concentration shown in the inset yields $\alpha K_{\text {gal, }}$ the dissociation constant for gallic acid in the presence of glucose. Assuming $K_{\text {gal }}=$ 1.73, then $\alpha=1.0$. (B) as in (A) except that the concentration of ellagic acid were $0(O), 5(O), 7(\square), 12(\square)$, 
and $15 \mu \mathrm{M}(\Delta)$.The replot of the slopes versus ellagic acid concentration shown in the inset yields treated as in (A) yields an interaction constant, $\alpha=0.22$.
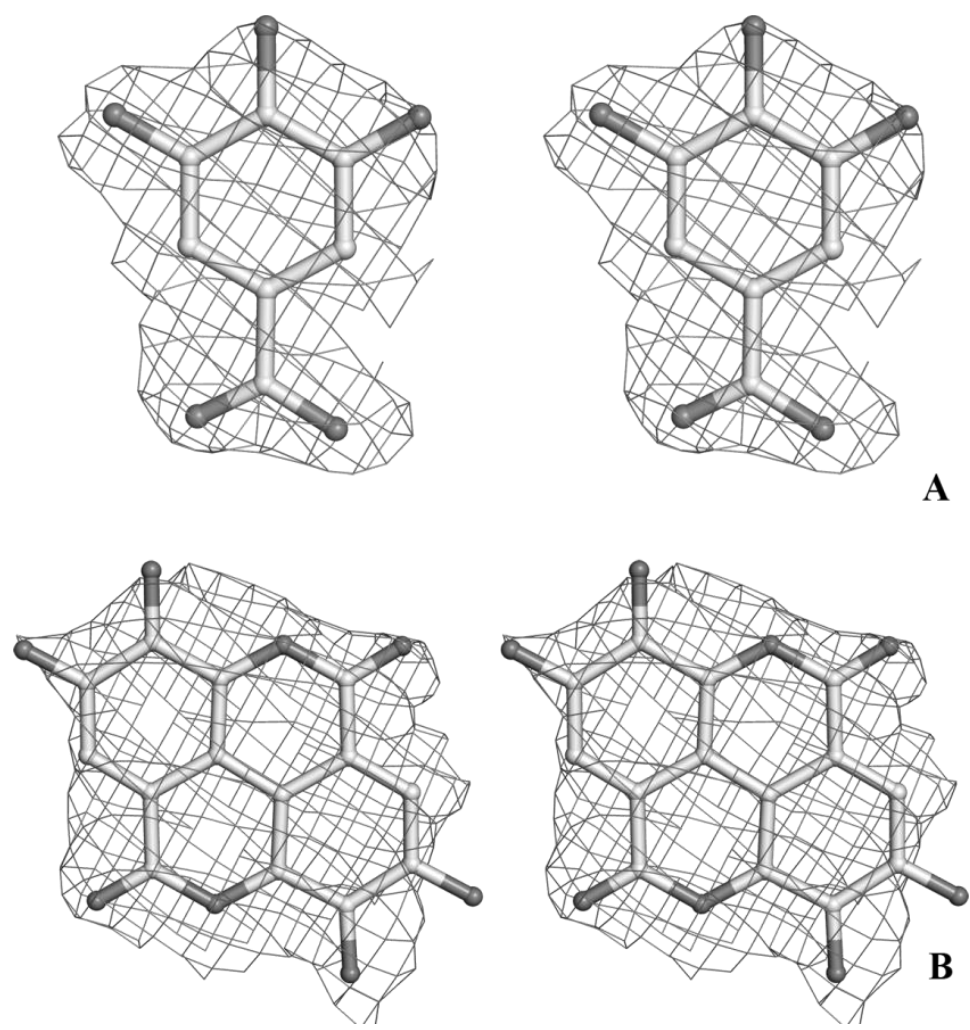

Figure 4. Stereo diagrams of the sigmaA $2 \mathrm{~F}_{\mathrm{O}}-\mathrm{F}_{\mathrm{C}}$ electron density map, contoured at $1 \sigma$, for the bound gallic (A) and ellagic (B) acid at GPb. Electron density maps were calculated before incorporating the inhibitor molecules in the refinement procedure. 

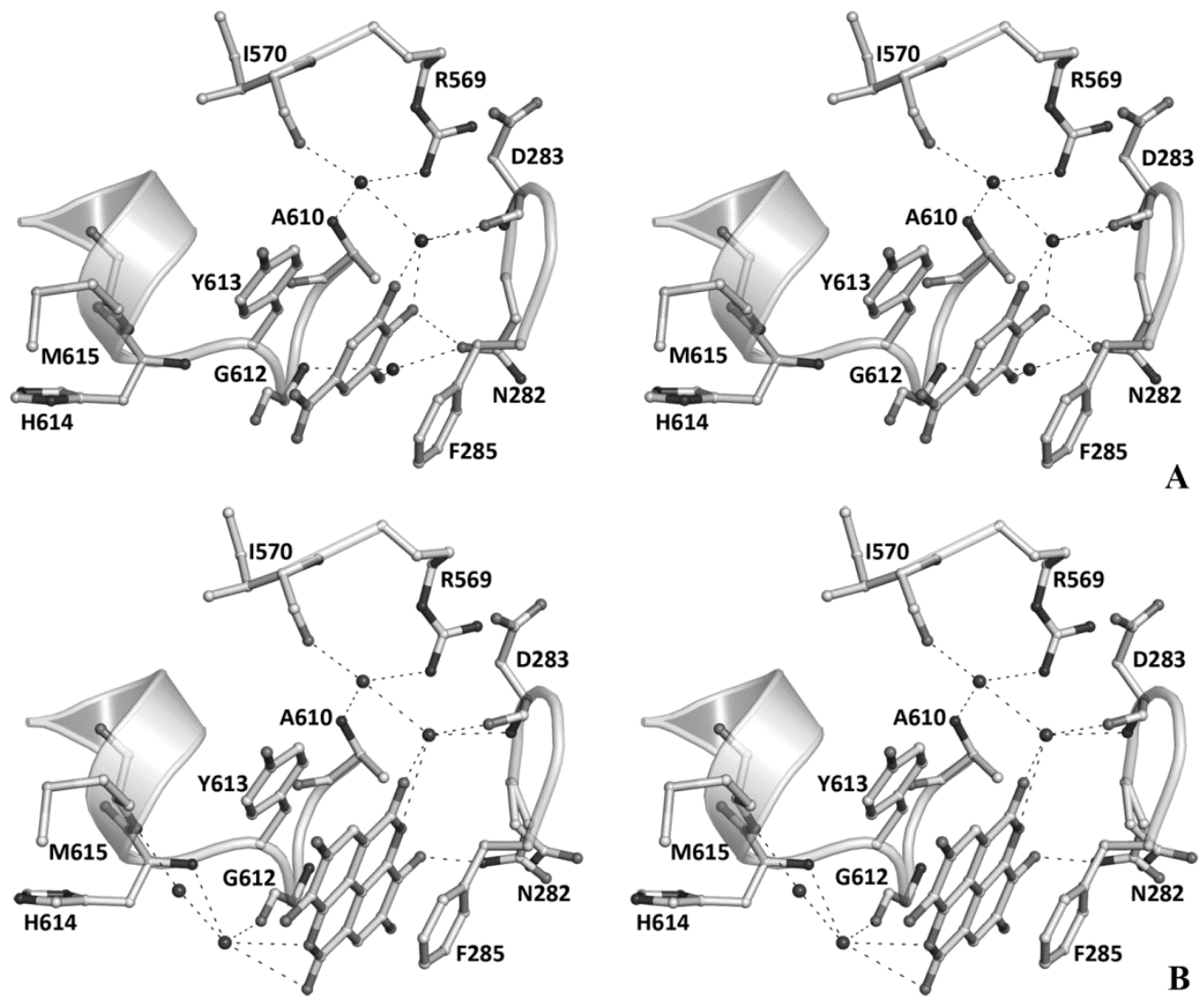

Figure 5. Stereo diagrams of the binding of gallic (A) and ellagic (B) acid to the inhibitor binding site of GPB. Hydrogen bond interactions are represented by dotted lines and water molecules as black spheres.
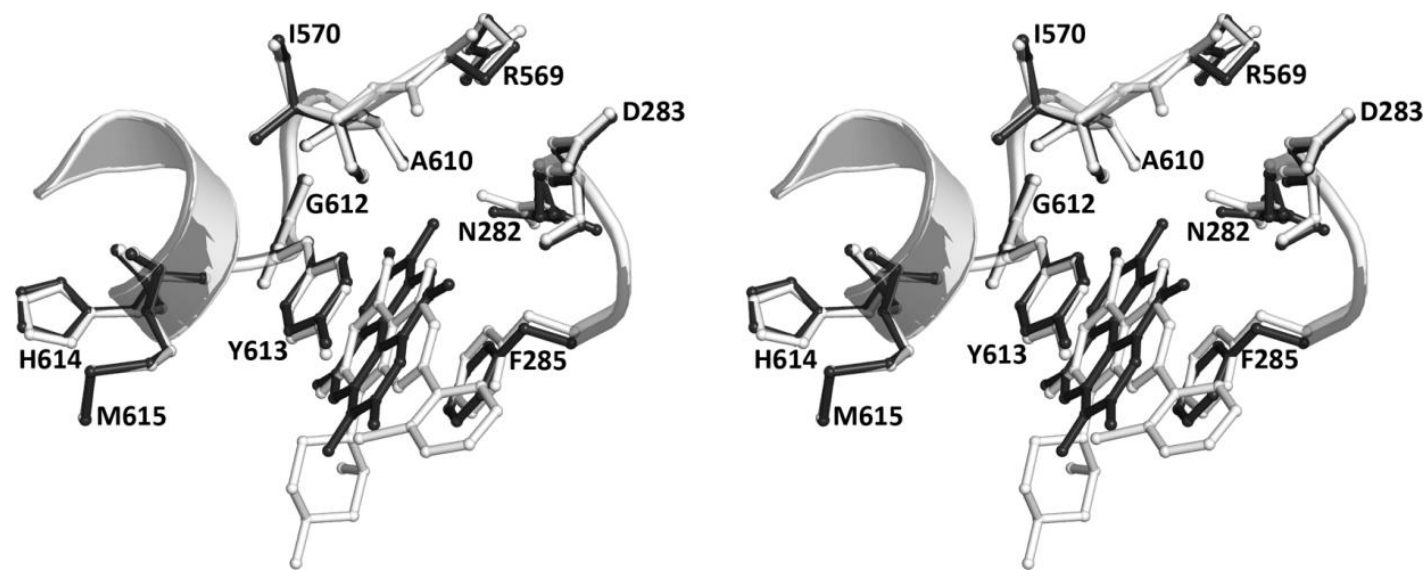

Figure 6. Structural comparison (in stereo) of the GPb-ellagic acid (black) and the GPb-flavopiridol (PDB entry: 1C8K; white) complexes in the vicinity of the inhibitor site. 